\title{
RADIAL FUNCTION BASED AB-INITIO TOMOGRAPHIC RECONSTRUCTION FOR CRYO ELECTRON MICROSCOPY
}

\author{
Yves Michels, Étienne Baudrier, Lö̈c Mazo \\ ICube, University of Strasbourg, CNRS \\ 300 Bd Sébastien Brant - CS 10413 - 67412 ILLKIRCH, FRANCE
}

\begin{abstract}
A cryo Electron Microscopy dataset is composed of tomographic projections of an object (e.g. a macromolecule). The projection orientation information is unknown. The scope of this paper is the tomographic reconstruction of the observed object in the ab-initio case where the volume has to be estimated only from a raw projection dataset. A new approach based on a parametric model of the volume is presented. The description of the model and the search of the parameters are detailed. The accuracy and robustness of the proposed reconstruction method is shown on synthetic and real databases.
\end{abstract}

Index Terms - tomography, cryo EM, parametrization, unknown directions

\section{INTRODUCTION}

In biology, the 3D structure of a protein plays an essential role in the determination of its biological functions. Cryo Electron Microscopy (Cryo EM) is one of the main imaging modalities used for macromolecule structure determination. It enables, after reconstruction to visualize the $3 \mathrm{D}$ electron density of large macro molecules (1,000 to 10,000 atoms). A Cryo EM dataset is composed of a large number (thousands to million) of images, each including a tomographic projection of the same molecule. Due to the acquisition principle, the projection direction (orientation) of each projection is unknown. Therefore, the construction of a 3D object from the Cryo EM dataset is a difficult problem with important biological consequences .

Because of the problem complexity, the computational cost of reconstruction methods is still an issue and can reach several hundreds of hours of CPU time for an ab-initio reconstruction. Recent softwares (SIMPLE [?], RELION [?] or Cryo-SPARC [?]) use massively parallelized implementations to reduce the execution time. Nevertheless, it is important to look forward the complexity reduction so as to treat bigger datasets (as heterogeneous datasets) or to reduce the computation time. We propose in this paper a method for abinitio reconstruction with a reduced number of operations that can be implemented in parallel.
The ab-initio reconstruction methods construct the density volume from a raw projection image dataset without prior volume information. Most of the ab-initio reconstructions involve two steps. In the first one, the orientations are estimated. Different approaches have been proposed in the literature: some are based on the common line property [?], some use non-linear dimension reduction to represent the projection images in a space where orientations can directly be estimated [?], and some use specific metrics as the moment based angular distance [?, ?]. In a second time, the volume is constructed. Several methods exist, using algebraic reconstruction [?], using iterative back propagation algorithms [?] or Fourier representation of the volume [?]. Other approaches propose to estimate jointly the orientations and the 3D volume. As in [?] where the construction is based on a cost optimization taking into account the common line property and the similarity between the projections of the estimated volume and the dataset. Facing the high computational cost of 3D computerized tomography, a reconstruction method based on a parametric model is presented in this study. The reduced number of parameters allows fast reconstruction resulting from an optimization.

The paper is structured as follows. In Section 2, the tomographic problem is defined and the reconstruction method is described. In Section 3, the implementation of our method including our optimization strategy is detailed and the evaluation of the proposed algorithm is given in Section 4. Finally, the conclusion of the study and some future works are given in the last section.

\section{RECONSTRUCTION ALGORITHM}

Let $\rho: \mathbb{R}^{3} \mapsto \mathbb{R}$ be a continuous function with compact support standing for the volume, and let $\mathcal{R} \in \mathrm{SO}(3)$, the orientation, be a rotation matrix. Let $\mathcal{P}_{\rho}(\mathcal{R})$ be the projection of $\rho$ for the orientation $\mathcal{R}$. The projection is given by the Radon transform with the formula:

$$
\mathcal{P}_{\rho}(\mathcal{R})(y, z)=\int_{-\infty}^{+\infty} \rho\left(\mathcal{R}^{-1}(x, y, z)\right) d x .
$$

We note $\pi$ the projection given by the sampling, possibly noisy, of $\mathcal{P}_{\rho}(\mathcal{R})$, and $m$ the width of the projection image 
(and also the sampling resolution). Let $\Pi$ be a finite set of sampled projections: $\Pi=\left\{\pi_{k}, k \in \llbracket 1, n_{p} \rrbracket\right\}$ where $n_{p}$ is the number of projections.

We propose to approximate the volume $\rho$ by a sum of radial Gaussians $\sum_{l=1}^{n_{g}} \rho_{l}$ where $n_{g}$ is the number of Gaussians and $\rho_{l}$ a radial Gaussian parametrized by its position, $X_{l}=\left(x_{l}, y_{l}, z_{l}\right)$, its width, $\delta_{l}$ and its intensity $\omega_{l}$ :

$$
\rho_{l}(x, y, z)=\frac{\omega_{l}}{\left(\sqrt{2 \pi} \delta_{l}\right)^{3} n_{g}} \exp \left(-\frac{R_{l}^{2}}{2 \delta_{l}^{2}}\right),
$$

where $R_{l}^{2}=\left(x-x_{l}\right)^{2}+\left(y-y_{l}\right)^{2}+\left(z-z_{l}\right)^{2}$. We note $\mathcal{S}\left(n_{g}\right)$ the set of the $n_{g} \times 5$ parameters of the Gaussian sum and $V\left(\mathcal{S}\left(n_{g}\right)\right)$ the Gaussian sum (abbreviated in $V$ if no ambiguity). Note that the linearity of the Radon transform implies that the projection of the volume $V$ is given by the sum of the projections of the Gaussians $\left(\rho_{l}\right)_{l}$. For a given orientation, $\mathcal{R}$, the projection of the volume $V$ is given analytically by:

$$
\mathcal{P}_{V}(\mathcal{R})(s, t)=\sum_{l=1}^{n_{g}} \frac{\omega_{l}}{2 \pi \delta_{l}^{2} n_{g}} \exp \left(-\frac{\left(s-s_{l}\right)^{2}+\left(t-t_{l}\right)^{2}}{2 \delta_{l}^{2}}\right)
$$

where $\left(r_{l}, s_{l}, t_{l}\right)^{t}=\mathcal{R} \times\left(x_{l}, y_{l}, z_{l}\right)^{t}$.

Note that we choose the Gaussian functions nevertheless other integrable spherical functions can also be used.

The tomographic reconstruction is performed by jointly estimating the volume parameters, $\hat{\mathcal{S}}$, and the orientation parameters, $\left\{\hat{\mathcal{R}}_{k}, k \in \llbracket 1, n_{p} \rrbracket\right\}$ that minimize cost function given by:

$$
E=\sum_{k=1}^{n_{p}} \sum_{i=1}^{m} \sum_{j=1}^{m}\left(\pi_{k, i, j}-\hat{\pi}_{k, i, j}\right)^{2}
$$

where $\pi_{k, i, j}$ is the $(i, j)$ pixel of the $k^{\text {th }}$ projection and $\hat{\pi}_{k}$ the sampled projection of $V\left(\hat{\mathcal{S}}\left(n_{g}\right)\right)$ with the estimated orientation $\hat{\mathcal{R}}_{k}$.

Intuitively, for a Gaussian number $n_{g}$ small against the voxel number, this approach is faster than a voxel based volume as the optimization is done on a smaller set of parameters, e.g. for a $256^{3}$ volume and $n_{g}=50$, there are 250 parameters for the proposed method in comparison to $256^{3}$ for a voxel based one. In addition, each estimated projection is computed in $O\left(\mathrm{~m}^{2}\right)$ instead of $O\left(\mathrm{~m}^{3}\right)$ when line integrals are computed.

The optimization implementation is done with a stochastic gradient descent; it is easily parallelizable and is adapted to a multi-scale approach. The detail of the optimization is given in the next Section.

\section{IMPLEMENTATION}

The parameter estimation is done alternatively on the orientations and the volume, as described in Algorithm 1. Both optimizations are described in the next subsections.

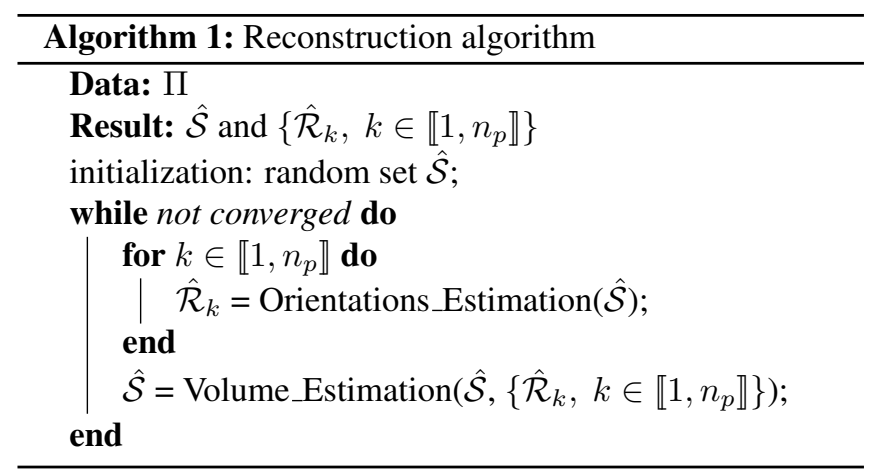

\subsection{Volume parameter estimation}

For a given set of orientations, $\left\{\hat{\mathcal{R}}_{k}, k \in \llbracket 1, n_{p} \rrbracket\right\}$, the volume parameters $\hat{\mathcal{S}}$ are computed performing a gradient descent. The optimization step is initialized with the last estimated parameters.

At each iteration, the gradient of $E$ is computed using the expressions given in Equations $(5,6,7)$.

$$
\begin{aligned}
\frac{\partial E}{\partial \omega_{l}}= & 2 \sum_{k=1}^{n_{p}} \sum_{i=1}^{m} \sum_{j=1}^{m}\left(\left(\hat{\pi}_{k, i, j}-\pi_{k, i, j}\right) \frac{1}{\omega_{l}} \hat{\pi}_{l, k, i, j}\right), \\
\frac{\partial E}{\partial \delta_{l}}=2 \sum_{k=1}^{n_{p}} \sum_{i=1}^{m} \sum_{j=1}^{m}\left(\left(\pi_{k, i, j}-\hat{\pi}_{k, i, j}\right) \times\right. & \left.\left(\frac{2}{\delta_{l}}-\frac{\left(s_{l, k}-s\right)^{2}+\left(t_{l, k}-t\right)^{2}}{\delta_{l}^{3}}\right) \hat{\pi}_{l, k, i, j}\right) \\
\frac{\partial E}{\partial X_{l}}= & 2 \sum_{i=1}^{m} \sum_{j=1}^{m}\left(\left(\pi_{k, i, j}-\hat{\pi}_{k, i, j}\right) \frac{\partial \hat{\pi}_{k, i, j}}{\partial X_{l}}\right),
\end{aligned}
$$

where

$$
\frac{\partial \hat{\pi}_{k, i, j}}{\partial X_{l}}=\hat{\mathcal{R}}_{k} \times\left(\begin{array}{c}
0 \\
s_{l, k}-s \\
t_{l, k}-t
\end{array}\right) \frac{1}{\delta_{l}^{2}} \hat{\pi}_{k, i, j} .
$$

The image $\hat{\pi}_{l, k}$ is the discrete projection of $V\left(\hat{\mathcal{S}}\left(n_{g}\right)\right)$ with the estimated orientation $\hat{\mathcal{R}}_{k}$.

The position, width and intensity parameters are updated independently, due to their heterogeneity. To be independent from the normalization of the projection dataset, the intensities are updated with a constant step and the positions and the widths are updated using a global constant norm:

$$
\begin{gathered}
\sum_{l=1}^{n_{g}}\left\|X_{l}^{(n)}-X_{l}^{(n+1)}\right\|_{2}^{2}=\lambda_{1}, \\
\sum_{l=1}^{n_{g}}\left(\delta_{l}^{(n)}-\delta_{l}^{(n+1)}\right)^{2}=\lambda_{2},
\end{gathered}
$$


where ${ }^{(n)}$ correspond to the $n^{\text {th }}$ gradient iteration. The fixed $\lambda_{1}$ and $\lambda_{2}$ parameters depend on the desired precision of the parameter estimation.

Note that our updating rules imply that passed a finite number of iterations, the resulting cost oscillate. Therefore, the volume optimization is terminated when the cost function stops decreasing.

Besides, to reduce computational cost and increase robustness to local minimums, each iteration is performed on a random subset of projections.

\subsection{Projection orientation estimation}

For a given volume parameter set, the search of the orientations is done independently for each projection. For a given projection, $\pi_{k}$, the cost in function of the orientation is not a convex function. For the tested objects presented in 4.1, the cost function may have several dozens of local minimums as shown in Figure 1.

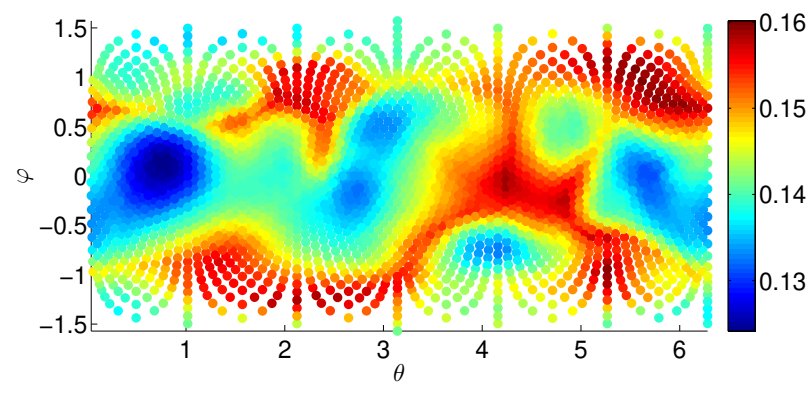

Fig. 1: Cost function for a projection from our real dataset in function of the angles $\theta$ and $\varphi$ (on the sphere). For each orientation on the sphere, the in plane rotation is chosen to minimize the cost function.

Orientations are pre-estimated by an exhaustive search on a fixed grid containing $162 \times 24$ orientations regularly sampled on $\mathrm{SO}(3)$ then refined by convex optimization.

Due to the stochastic gradient descent, the whole optimization converge in a local minimum. Nevertheless only the resulting volume, $V\left(\hat{\mathcal{S}}\left(n_{g}\right)\right)$, is important, not the parameter set, $\hat{\mathcal{S}}\left(n_{g}\right)$. In addition, empirical evaluations show that the volume converges to an acceptable optimum that allows the orientations to converge to the global optimum.

\section{EVALUATION}

The reconstruction algorithm is performed on synthetic and real datasets to evaluate its ability to reconstruct finely the volume and to estimate the projection orientations.

\subsection{Description of our datasets}

Synthetic datasets are generated from Protein Data Bank files (PDB) where the centroids of the atoms which constitute the protein are given. The volume is generated with a sum of bounded Gaussians:

$$
\rho^{a}(x)=\exp \left(1-1 /\left(1-\frac{\|x\|^{2}}{d_{a}^{2}}\right)\right),
$$

where $d_{a}$ is the average size of the amino acids. To reduce the complexity of the volume, each Gaussian is centered on amino acid centroids. An example of volume is shown in Figure 2.

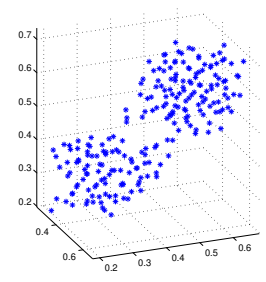

(a) Amino acid centroids

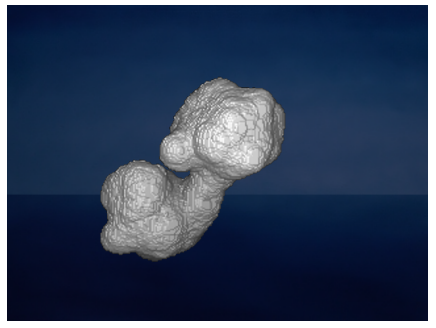

(b) Synthetic 3D volume
Fig. 2: Synthetic dataset generated from a PDB.

Our synthetic dataset is composed of 7 datasets of 5,000 projections with resolution $128 \times 128$. The datasets are generated from different proteins. Noisy dataset are noised by additive white Gaussian noise with the desired signal-to-noise ratio (SNR).

The real dataset is composed of 1,000 projections from the TAF7 (TAFII55 complex), with resolution $160 \times 160$, averaged and corrected according to the contrast transfer function. Examples of projection images from different datasets are shown in Figure 3.

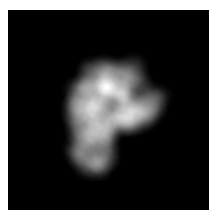

(a) $\mathrm{SNR}+\infty$

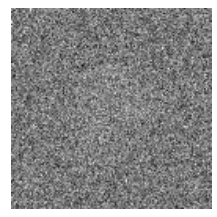

(b) SNR 1/64

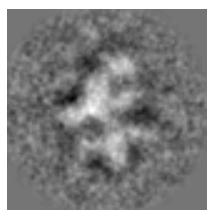

(c) Real projection
Fig. 3: Example of projection images. (a) synthetic projection, (b) noisy realization of (a), (c) averaged projection from TAF7.

\subsection{Results}

The reconstructions are evaluated on the volume correlations:

$$
\frac{\langle\rho \mid V\rangle}{\|\rho\| \mid\|V\|},
$$


where $\langle\cdot \mid \cdot\rangle$ is the canonical scalar product. The estimated orientations are evaluated with the average angular difference between estimated and true orientations.

Two series of tests were performed, one to evaluate the ability to reconstruct finely the volume in the noiseless case, and one to evaluate the robustness of the reconstruction algorithm to the noise. The number of Gaussians used for the reconstruction are respectively 115 Gaussians and 35 Gaussians for the dataset with SNR $+\infty$ and SNR 1/64. The results are given in Table 1

\begin{tabular}{|c|c|c|c|c|}
\hline $\mathrm{n}$ & $\begin{array}{c}\text { Volume } \\
\text { Corr. }\end{array}$ & $\begin{array}{c}\text { angular } \\
\text { difference (rad) }\end{array}$ & $\begin{array}{c}\text { Volume } \\
\text { Corr. }\end{array}$ & $\begin{array}{c}\text { angular } \\
\text { difference (rad) }\end{array}$ \\
\hline & \multicolumn{2}{|c|}{ SNR $+\infty$} & \multicolumn{2}{|c|}{ SNR 1/64 } \\
\hline 1 & 0.9966 & $0.0368 \pm 0.1326$ & 0.9554 & $0.2700 \pm 0.0458$ \\
2 & 0.9662 & $0.0106 \pm 0.0065$ & 0.9014 & $0.0275 \pm 0.0168$ \\
3 & 0.9762 & $0.0137 \pm 0.0076$ & 0.9587 & $0.0200 \pm 0.0115$ \\
4 & 0.9663 & $0.0139 \pm 0.0087$ & 0.8182 & $0.5249 \pm 0.9615$ \\
5 & 0.9820 & $0.0147 \pm 0.0079$ & 0.9329 & $0.0500 \pm 0.0345$ \\
6 & 0.9903 & $0.0122 \pm 0.0064$ & 0.9413 & $0.0224 \pm 0.0123$ \\
7 & 0.9966 & $0.0190 \pm 0.0104$ & 0.9703 & $0.0334 \pm 0.0241$ \\
\hline
\end{tabular}

Table 1: Reconstruction evaluation for synthetic datasets.

The volume correlations in the noise free case are close to one. Therefore, our proposed method is able to reconstruct finely the volumes. In addition, the estimated orientation error is smaller than $0.02 \mathrm{rad}(1.15 \mathrm{deg})$, except the first volume where 13 orientations error are higher than $1 \mathrm{rad}$ due to the quasi symmetry of the volume.

The results in Table 1 shows the robustness of our method to the noise, even with SNR $1 / 64(-18 \mathrm{~dB})$. Indeed, the constructed volumes have correlations higher than 0.9 except for one reconstruction. For totally asymmetric volumes, the orientation error is smaller than $0.05 \mathrm{rad}$.

Our reconstructed volumes have been compared to the ones obtained by Cryo-SPARC for the 7 noisy datasets (SNR $1 / 64)$. The average correlation and angular difference are respectively 0.7881 and $0.7479 \mathrm{rad}$, in comparison to 0.9255 and 0.1355 rad for our reconstruction method. The estimated volume and orientations are more accurate for our ab-initio reconstruction that allow faster post processing refinement.

The reconstruction from real dataset is geometrically similar to a reference $a b$-initio volume obtained with a tilt acquisition as shown in Figure 4. Note that the reference volume is not finely reconstructed therefore the correlation coefficient is 0.858 .

A test on $128^{3}$ volumes where the number of Gaussians $n_{g}$ is set to 50 and $n_{p}=1.7 \times 128$, gives reconstruction times for our method more than ten times faster than the integral based reconstruction method of [?].

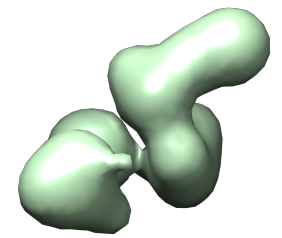

(a) Our reconstructed volume

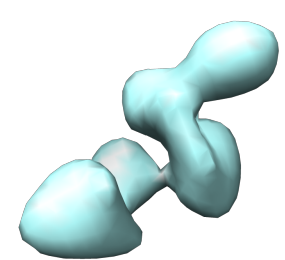

(b) Reference volume
Fig. 4: Reconstructed volumes.

\section{CONCLUSION}

This paper present a new 3D tomographic reconstruction method, based on a parametric model, that estimates jointly the volume of the observed object and the orientations of the projection dataset. The reconstruction is performed by optimizing the orientations and the model parameters to minimize a global cost function.

Due to its low number of parameters in comparison to the number of voxels in the desired 3D volume, our approach allows fast reconstruction even from very noisy dataset (SNR $1 / 64$ for $128 \times 128$ projection images).

The existing demonstration algorithm will be developed in $\mathrm{C}++$ and parallelized for GPU implementation. The optimal translation search will be added to the orientation estimation to improve the robustness of the reconstruction on real Cryo Electron Microscopy datasets.

This method will be extended to heterogeneous datasets, arising from deformable volumes, where the deformations can be discrete or continuous.

\section{REFERENCES}

[1] D. Elmlund and H. Elmlund, "SIMPLE: Software for ab initio reconstruction of heterogeneous single-particles," J. Struct. Biol., vol. 180, no. 3, pp. 420-427, 2012.

[2] S. H. W. Scheres, "RELION: Implementation of a Bayesian approach to cryo-EM structure determination," J. Struct. Biol., vol. 180, no. 3, pp. 519-530, 2012.

[3] A. Punjani, J. L. Rubinstein, D. J. Fleet, and M. A. Brubaker, "cryoSPARC: algorithms for rapid unsupervised cryo-EM structure determination," Nat. Methods, vol. 14, no. 3, pp. 290-296, 2017.

[4] M. Cohen, Y. Shkolnisky, and A. Yeredor, "Collaborative Detection Of Common Lines In Cryo EM Images Using Maximum Likelihood," in IEEE Conv. Electr. Electron. Isr., 2014, pp. 1-5. 
[5] R. R. Coifman, Y. Shkolnisky, F. J. Sigworth, and A. Singer, "Graph Laplacian tomography from unknown random projections." IEEE Trans. Image Process., vol. 17, no. 10, pp. 1891-1899, 2008.

[6] D. Salzman, "A method of general moments for orienting 2D projections of unknown $\{3 \mathrm{D}\}$ objects," Comput. Vis., Graph., Image Process., vol. 50, pp. 129-156, 1990.

[7] M. S. Phan, E. Baudrier, L. Mazo, and M. Tajine, "Estimation of Angular Difference Between Tomographic Projections Taken at Unknown Directions in 3D," Int. Conf. Image Process., 2015.

[8] X. Wan, F. Zhang, and Z. Liu, "Modified simultaneous algebraic reconstruction technique and its parallelization in cryo-electron tomography," in IEEE Proc. Int. Conf. Parallel Distrib. Syst., no. i, 2009, pp. 384-390.

[9] M. T. Mccann and M. Unser, "High-Quality ParallelRay X-Ray CT Back Projection Using Optimized Interpolation,' IEEE Trans. Image Process., vol. 26, no. 10, pp. 4639-4647, 2017.

[10] S. Swerdloff, K. Dabbs, X. Wang, and J. Holden, "Iterative reconstruction of tomographic images with fourier components as model parameters," in IEEE Int. Conf. Image Process., 1994, pp. 0-3.

[11] B. Ben Cheikh, É. Baudrier, and G. Frey, “Joint Direction And Volume Tomographical Ab Initio Reconstruction For Electron Microscopy," in Int. Symp. Biomed. Imaging, 2015, pp. 1040-1043. 